\title{
Immunoglobulin G4-related Lung Disease Accompanied by Organizing Pneumonia
}

\author{
Hiroki Suzuki ${ }^{1}$, Mari Watanabe ${ }^{1}$, Tomoka Ara ${ }^{1}$, Midori Nishizuka ${ }^{1}$, \\ Mami Morita $^{1}$, Chisa Sato ${ }^{1}$, Fumihiro Tsuchida ${ }^{1}$, Hiroaki Takeda ${ }^{1}$, \\ Nobukazu Tomichi ${ }^{2}$ and Yoshinori Kawabata ${ }^{3}$
}

\begin{abstract}
The full picture of immunoglobulin G4-related lung disease (IgG4-RLD) has not yet been elucidated. A 69-year-old man was referred to us with a more than 2-week history of productive cough and fatigue. Chest CT showed an airspace consolidation along the bronchovascular bundles. The pathological findings that were obtained from an open-lung biopsy showed both organizing pneumonia and interstitial pneumonia. Based on the established, comprehensive diagnostic criteria for IgG4-related disease (RD) as of 2011, this patient was given a definitive diagnosis of IgG4-RD. A further accumulation and analysis of those cases that concomitantly present with both IgG4-RLD and organizing pneumonia, like our patient, may contribute to the elucidation of the pathology of IgG4-RLD and the establishment of the disease spectrum.
\end{abstract}

Key words: IgG4-related lung disease, organizing pneumonia, interstitial pneumonia, chronic eosinophilic pneumonia

(Intern Med 52: 2105-2111, 2013)

(DOI: 10.2169/internalmedicine.52.0112)

\section{Introduction}

In recent years, much attention has been given to immunoglobulin G4 (IgG4)-related disease (IgG4-RD), which is characterized by high serum IgG4 levels as well as the infiltration of numerous IgG4-positive plasma cells and fibrosis in target organs. Although interstitial pneumonia and inflammatory pseudotumor are well known to be on the IgG4related lung disease (RLD) spectrum, there are only a few reports of IgG4-RLD, and the clinical and pathological details have yet to be examined (1-7). Thus, we suspect that some cases of IgG4-RLD alone, i.e., those cases which exhibit no extra-pulmonary lesions, may have been misdiagnosed as other diseases.

We encountered a case of IgG4-RLD presenting with the pathological findings of interstitial pneumonia associated with concomitant organizing pneumonia. We herein report the findings of this case, given the apparent importance of documenting the clinical features for the purpose of elucidating the pathology of IgG4-RLD and establishing the disease spectrum.

\section{Case Report}

A 69-year-old man experienced dyspnea while clearing snow in mid-February of 2009. In early March, a productive cough and fatigability appeared. In mid-March, he visited a neighborhood clinic where chest radiography revealed abnormal shadows. He was thus referred and admitted to our department for a detailed examination. Starting at 65 years of age, he had been treated for bronchial asthma, chronic sinusitis, atrial fibrillation and hypertension. At 66 years of age, he was admitted to a neighborhood hospital with a diagnosis of atrial fibrillation and cardiac failure. He had no history of allergic rhinitis or atopic dermatitis. He had smoked 30 cigarettes per day, from 20 years of age to 65 years of age. He had worked in the dyeing industry until he

\footnotetext{
${ }^{1}$ Department of Respiratory Medicine, Saiseikai Yamagata Saisei Hospital, Japan, ${ }^{2}$ Department of Pathology, Iwate Prefectural Central Hospital, Japan and ${ }^{3}$ Division of Diagnostic Pathology, Saitama Cardiovascular and Respiratory Center, Japan

Received for publication January 15, 2013; Accepted for publication April 21, 2013

Correspondence to Dr. Hiroki Suzuki, hiroki-s@ma.catvy.ne.jp
} 


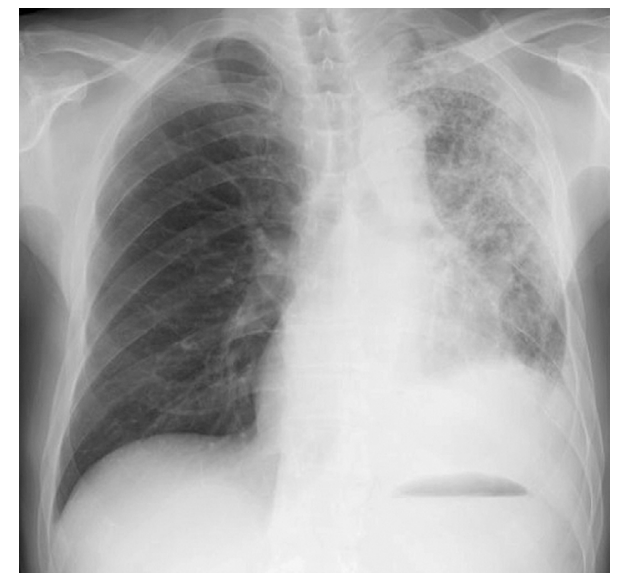

Figure 1. Chest radiography at the first visit demonstrates the shrinkage of the left lung by the infiltrative shadows in the left whole lung field and left pleural effusion.

was 66 years of age. At the time of his admission, he was receiving candesartan, warfarin potassium, bisoprolol fumarate, cilnidipine, spironolactone and a salmeterol xinafoate/fluticasone propionate combination product.

\section{Physical findings}

The physical findings were as follows: body temperature, $36.9^{\circ} \mathrm{C}$; blood pressure, $112 / 72 \mathrm{mmHg}$; pulse, irregular at 108 beats/min; oxygen saturation, $93 \%$ (room air); palpebral conjunctiva, not anemic; bulbar conjunctiva, not icteric; proptosis, absent; swelling of the parotid/submandibular glands, absent; superficial lymph nodes, not palpable; coarse crackles and rales, audible in the left precordia; cardiac murmurs, absent; abnormalities of the abdomen, absent and joint deformation, absent.

\section{Laboratory results}

The white blood cell (WBC) count was 7,300 cells/ $\mu \mathrm{L}$. In the WBC fraction, the proportion of eosinophils was elevated to $14.9 \%$. The hemoglobin, total protein and albumin levels were $11.6 \mathrm{~g} / \mathrm{dL}, 7.4 \mathrm{~g} / \mathrm{dL}$ and $3.3 \mathrm{~g} / \mathrm{dL}$, respectively, thus revealing mild anemia and hypoalbuminemia. The lactate dehydrogenase, C-reactive protein, immunoglobulin (Ig) $\mathrm{G}$ and $\mathrm{IgE}$ levels were $268 \mathrm{IU} / \mathrm{L}, 3.90 \mathrm{mg} / \mathrm{dL}, 2,077 \mathrm{mg} / \mathrm{dL}$ and $320 \mathrm{IU} / \mathrm{mL}$, respectively, where all four values are slightly above normal. The patient was negative for antinuclear antibody, anti-Sjogren's syndrome (SS)-A antibody, anti-SS-B antibody, myeloperoxidase (MPO)-specific antineutrophil cytoplasmic antibody (ANCA), proteinase 3 (PR3)-ANCA and angiotensin-converting enzyme (ACE). There were no decreases in the complement components (CH50 and C3). The rheumatoid factor was positive at 110 U/mL. While the Krebs von den Lungen-6 (KL-6) level was $338 \mathrm{U} / \mathrm{mL}$, the surfactant protein-D (SP-D) level was elevated $208 \mathrm{ng} / \mathrm{mL}$. A urinalysis revealed no abnormalities. Streptococcus pneumoniae was cultured from the sputum, but no growth of acid-fast bacteria was detected. The pulmonary function test results were normal, with a vital capac- ity $82.5 \%$ of predicted, a forced expiratory volume $1.0 \%$ (FEV1.0\%) of $75.7 \%$ and a diffusion lung capacity for carbon monoxide (DLCO) of $91.8 \%$ of predicted. Chest radiography showed infiltrative shadows in the entire left lung field and left pleural effusion (Fig. 1).

\section{Chest computed tomography (CT) (Fig. 2A-D)}

Infiltrative shadows were observed along the bronchovascular bundles of the left lung, and scattered infiltrative shadows reflecting emphysematous changes were observed around these bundles. Within these infiltrative shadows, traction bronchiectasis was observed. Bilateral hilar and mediastinal lymphadenopathy as well as a pleural effusion on the left side were also observed. Mild infiltrative shadows were present directly under the pleura of the right upper lobe.

On Hospital Day 5, chest CT revealed that the infiltrative shadows directly under the pleura of the right lung were becoming worse (Fig. 3A). Aspiration of the pleural effusion revealed an increase in the lymphocytes to $61.0 \%$. Furthermore, this exudative pleural effusion was mainly composed of lymphocytes. Although fluorine-18-2-fluoro-2-deoxy-Dglucose (FDG) positron-emission tomography (PET) revealed an intense FDG accumulation corresponding to the infiltrative shadows in both lungs and a mild to moderate FDG accumulation in the hilar and mediastinal lymph nodes, there was no FDG accumulation in any other organs, such as the pancreas. Bronchoscopy revealed no abnormalities within the visible range. Although the bronchoalveolar lavage results were provided as a reference due to the low recovery rate of $38.0 \%$, the proportions of lymphocytes, neutrophils and eosinophils were all observed to be elevated at $41.0 \%, 26.0 \%$ and $31.0 \%$, respectively. The CD4/CD8 ratio was 0.58 . Because the histopathological examination of the transbronchial lung biopsy samples (left $\mathrm{B}^{1+2} \mathrm{c}$, left $\mathrm{S}^{3} \mathrm{a}$ and left $\mathrm{S}^{3} \mathrm{c}$ ) revealed only a non-specific fibrotic thickening of the alveolar septa and an infiltration of inflammatory cells, an open lung biopsy (left $S^{1+2}$ and left $S^{6}$ ) was performed in early April of 2009. The histopathological findings were similar in these two lung tissue samples. Although various changes were observed in these tissues, one was considered to be indicative of organizing pneumonia, while the other suggested a diagnosis of interstitial pneumonia. In the organizing pneumonia lesion (Fig. 4A, B), the fibrotic granulation tissue that is typically associated with the infiltration of numerous inflammatory cells into alveoli and alveolar ducts was frequent. In the surrounding alveolar space, there were several histiocytes, a few foreign body giant cells, lymphocytes, plasma cells, etc. Furthermore, regions infiltrated by dense aggregates of eosinophils were observed in some alveolar spaces, and an eosinophilic abscess-like lesion (Fig. 4C) was also observed an one area where the histiocytes had increased in number as they had engulfed the degenerated eosinophils. In the interstitial pneumonia lesion (Fig. 4D, E), the alveolar septa were diffusely thickened due to a marked infiltration of inflammatory cells, such as plasma cells and lymphocytes. Concurrently, numerous in- 


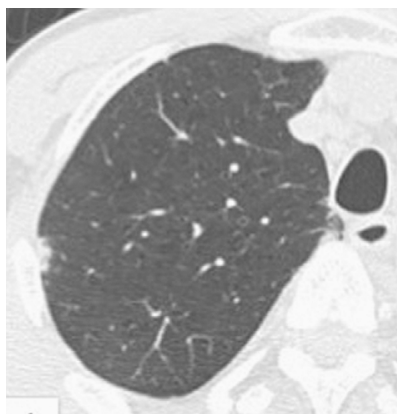

A

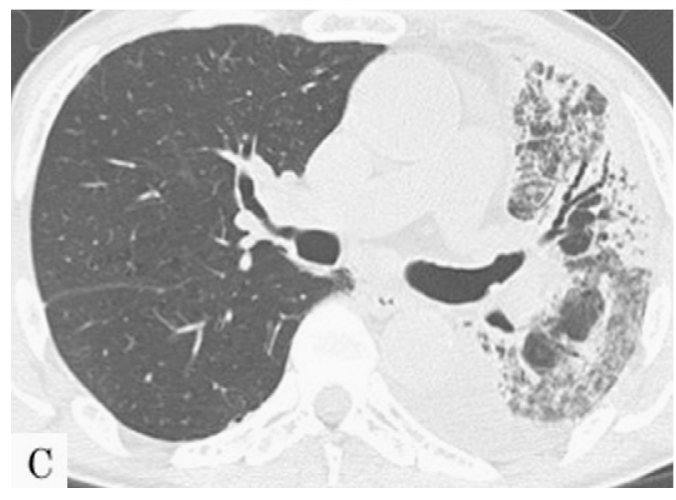

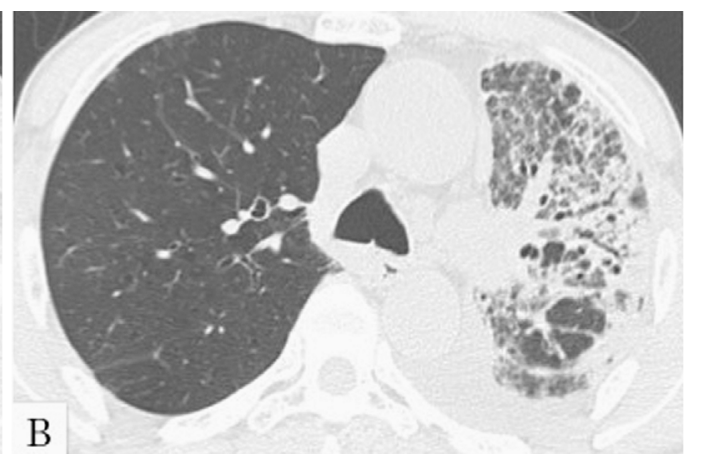

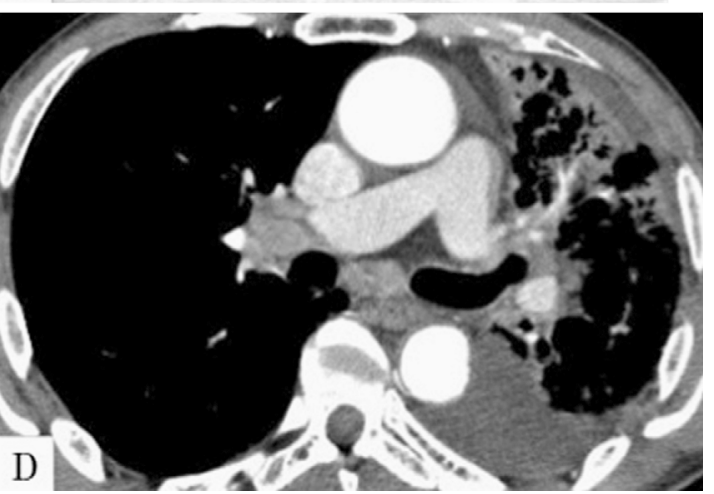

Figure 2. Chest CT scan at the first visit demonstrates airspace consolidation along bronchovascular bundles with traction bronchiectasis, peripherally accompanied by scattered foci of consolidation reflecting emphysema in the left lung. Swelling of the bilateral hilar and mediastinal lymph nodes and left pleural effusion are seen (A-D). Minimal consolidation is also noted in the subpleural lesion of the right upper lobe (A).
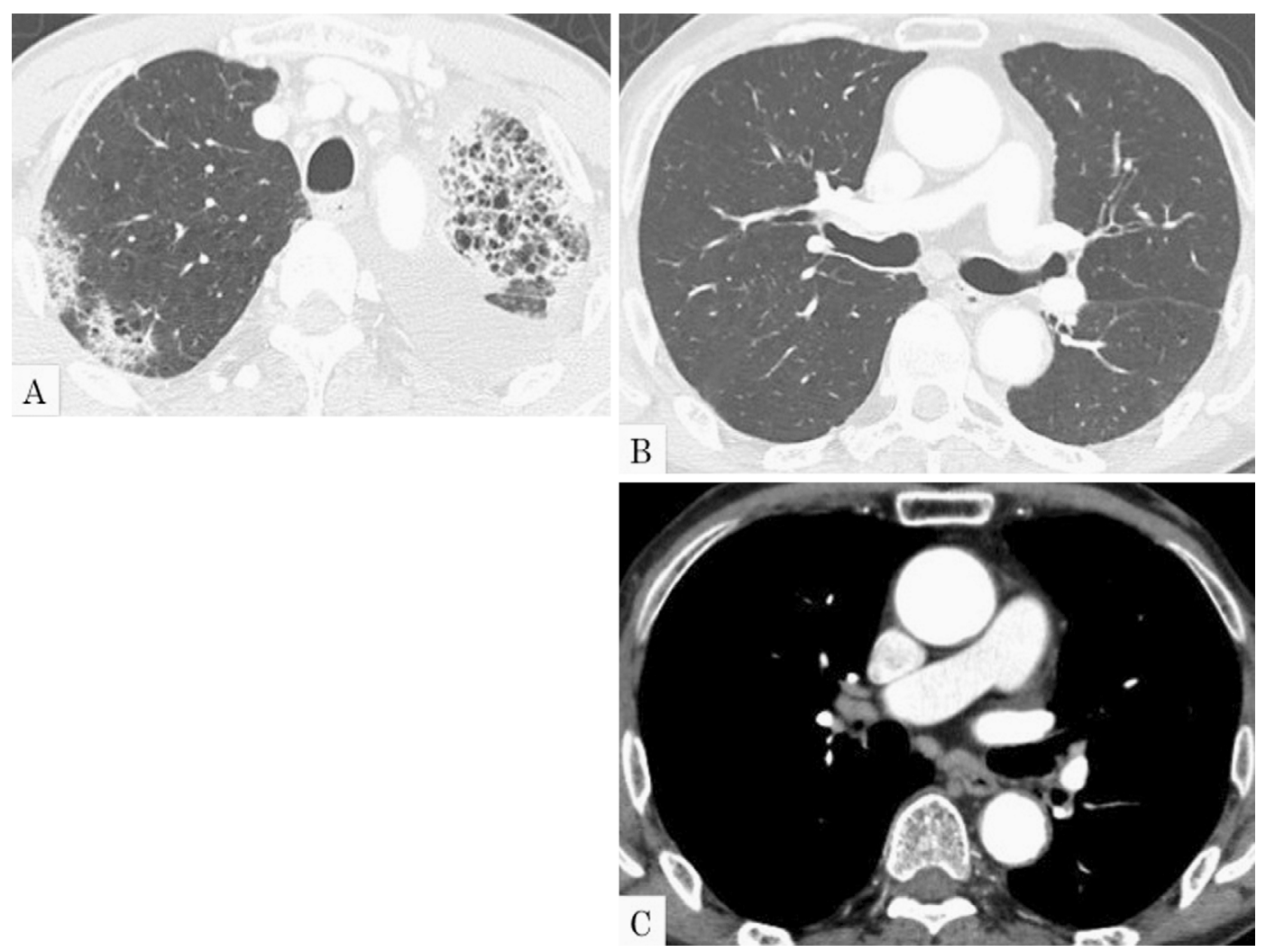

Figure 3. On Hospital Day 5, chest CT revealed worsening of infiltrative shadows directly under the pleura of the right lung (A). Two years later, chest CT confirmed disappearance of these lesions in both lung fields and reductions in the sizes of the hilar and mediastinal lymph nodes $(\mathrm{B}, \mathrm{C})$. 

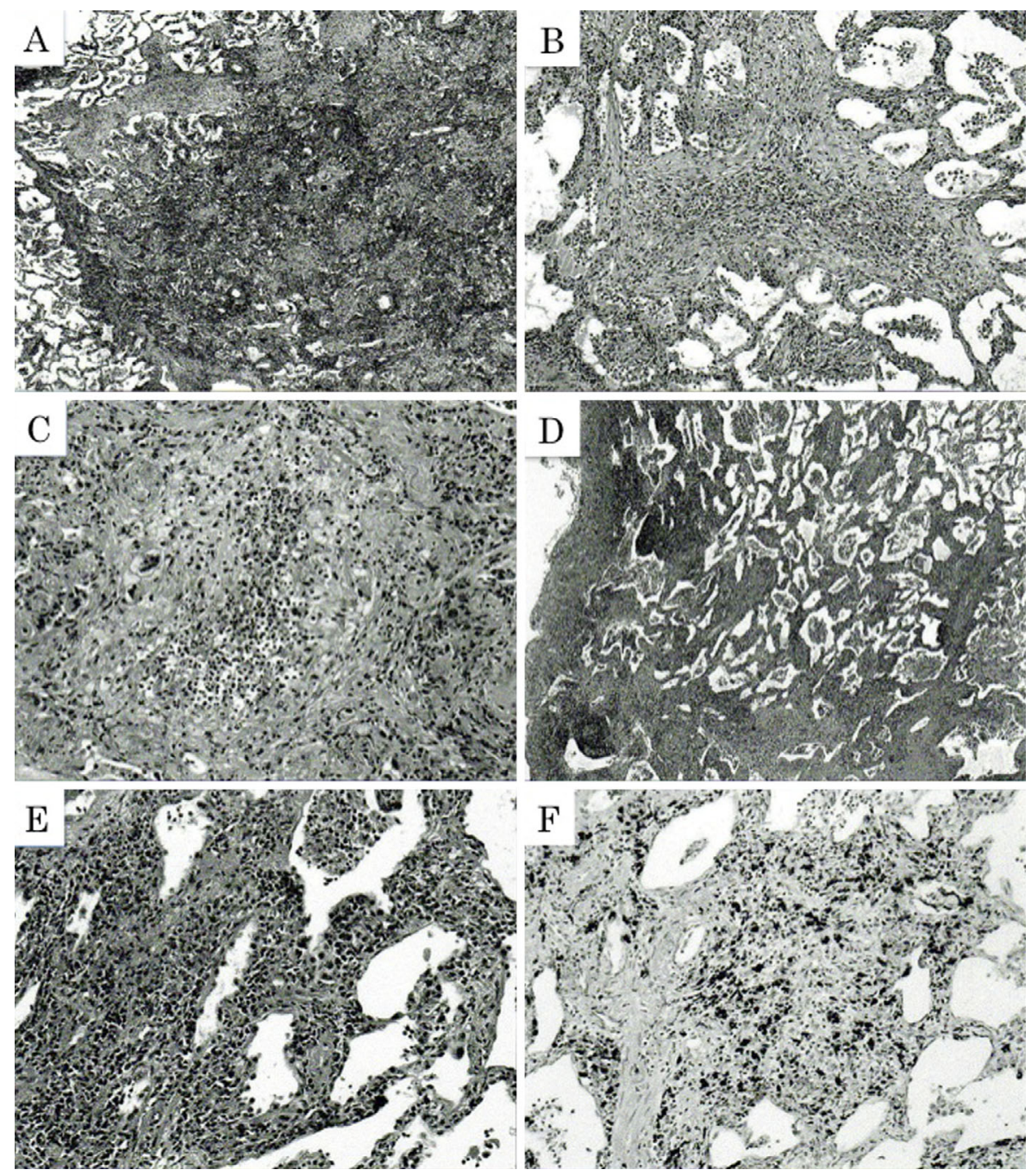

Figure 4. Histopathological examination of open lung specimen from left S1+2. Organizing pneumonia is evident. [A: Elastica Masson Goldner (EMG) staining, low-power field. B: Hematoxylin and Eosin staining (H\&E), midpower field.] The alveoli and alveolar ducts are filled with fibrous granulation tissue accompanied by numerous inflammatory cells. Moreover, many macrophages and inflammatory cells are visible in alveolar spaces. Eosinophilic abscess-like change is noted. (C: H\&E, high-power field.) Interstitial pneumonia is evident. (D: H\&E, low-power field, E: H\&E, highpower field.) Diffuse alveolitis with thickening of the alveolar septa is accompanied by marked lympho-plasmacytic infiltration. Numerous IgG4-positive plasma cells are identifiable in the alveolar interstitium. (F: IgG4 immunostaining, mid-power field.) IgG4 positive plasma cells accounted for $68.3 \%$ of IgG positive plasma cells.

flammatory cells had infiltrated the bronchiolar wall, peribronchiolar interstitium, perivascular interstitium, subpleural interstitium and pleura. Furthermore, the formation of an aggregated lymphoid lesion and lymphoid follicles was observed in a portion of the lesion. However, there was little structural destruction of the lung. As for vascular changes, intimal thickening of the small vessels and capillaries was observed, but neither occluded vascular lumens nor signs of vasculitis were detected. In this patient, because the infiltration of plasma cells was prominent in not only the alveolar septa but also broadly in the interstitium, various Ig-positive cells were immunohistologically analyzed (using 5 visual fields with a $\times 40$ objective lens). The Ig-positive cells that had infiltrated the pulmonary interstitium were as follows:
IgG-positive cells, 187.6 cells/high power field (HPF) (147226 cells/HPF); IgG4-positive cells (Fig. 4F), 128.2 cells/ HPF (107-149 cells/HPF); IgA-positive cells, 1.2 cells/HPF (0-5 cells/HPF) and IgM-positive cells, 11.2 cells/HPF (6-15 cells/HPF). The IgG4: IgG ratio was $68.3 \%$. Light chain restriction was not observed. The IgG4 level as measured in a serum sample that was obtained in early April was elevated at $250 \mathrm{mg} / \mathrm{dL}$. Thus, this patient was diagnosed as having IgG4-RLD.

Following the open lung biopsy, the shadows in the left lung resolved spontaneously, but those in the right lung continued to worsen. In mid-April, the oral administration of prednisolone (PSL) was started at a dose of $40 \mathrm{mg} / \mathrm{day}$. Although the dose was subsequently tapered, the abnormal 

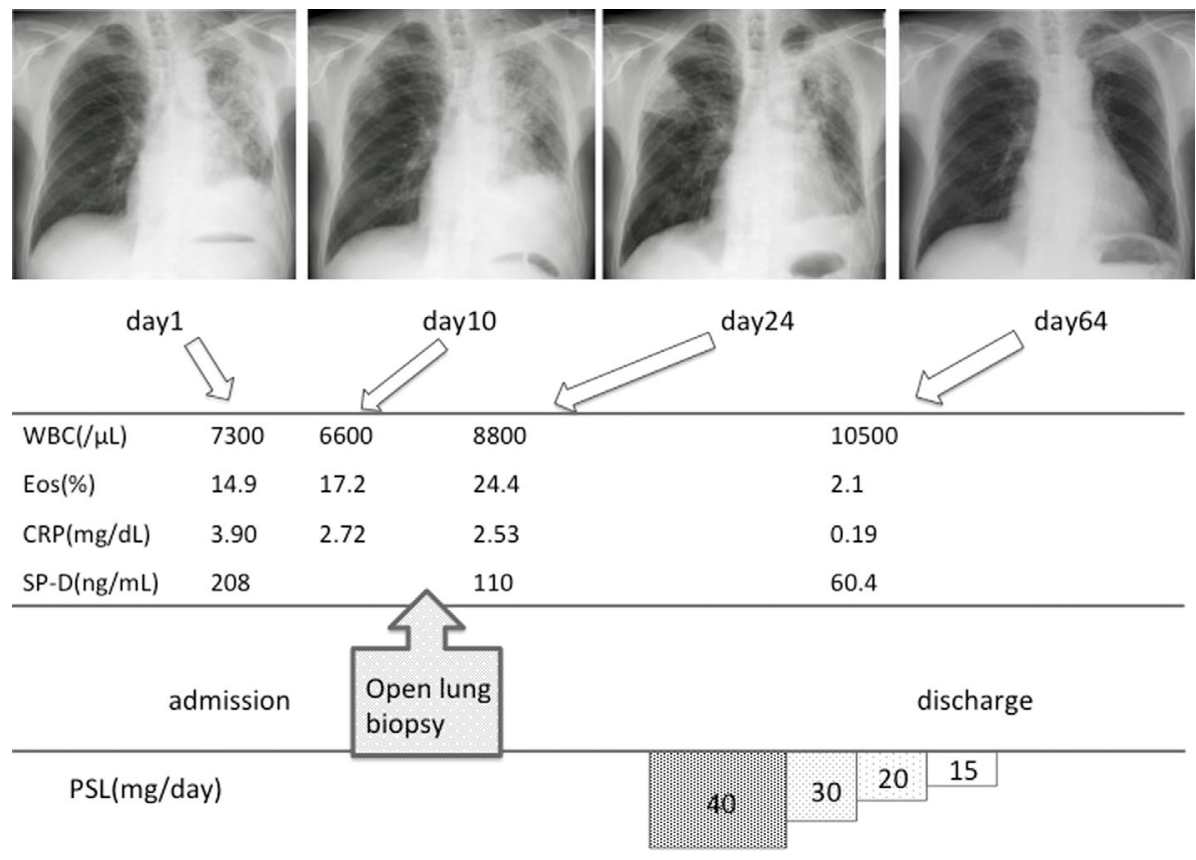

Figure 5. Clinical course.

shadows on chest radiography nearly disappeared within one month (Fig. 5). Since his discharge in mid-May, the patient has been followed up at our outpatient clinic. He has been receiving PSL at a maintenance dose of $5 \mathrm{mg}$ /day since August and no signs of relapse have been observed to date. On chest CT, the disappearance of the lesions in the lung fields and a reduction in the sizes of the hilar and mediastinal lymph nodes was confirmed at 2 years after the initial diagnosis (Fig. 3B, C).

\section{Discussion}

The reported patient satisfied all three of the comprehensive diagnostic criteria for IgG4-RD as of 2011 (8): a clinical examination showing characteristic diffuse/localized swelling or masses in either single or multiple organs; a hematological examination showing elevated serum IgG4 concentrations ( $\geq 135 \mathrm{mg} / \mathrm{dL})$ and a histopathological examination showing (a) marked lymphocyte and plasmacyte infiltration and fibrosis and (b) infiltration of $\mathrm{IgG}_{4}^{+}$plasma cells, a $\mathrm{IgG}^{+} / \mathrm{IgG}^{+}$cell ratio $>40 \%$ and $>10 \mathrm{IgG}^{+}$plasma cells/ HPF. Thus, a definitive diagnosis of IgG4-RD was made. However, it was highly important to exclude multicentric Castleman's disease (MCD) from the differential diagnosis because he did not exhibit the extra-pulmonary lesions that are generally consistent with IgG4-RD, rather, he showed a high interleukin (IL)-6 level of $14.2 \mathrm{pg} / \mathrm{mL}$ in a cryopreserved serum sample even though he did not undergo either a hilar or a mediastinal lymph node biopsy. In this patient, the diagnosis of IgG-RD was more reasonable than a diagnosis of MCD for the following reasons: an advanced onset age; the presence of mild anemia (9); a histologicallyconfirmed IgG4-rich inflammation (10); a histologicallyprominent eosinophil infiltration (11) and the achievement of a complete response to the steroid therapy followed by a long period without recurrence or exacerbation.

Inoue et al. reported the CT findings of $13 \mathrm{IgG} 4-\mathrm{RLD}$ patients identifiable by 10 radiologic findings, including ground-glass opacity (GGO), nodules and the thickening of the bronchovascular bundles and the interlobular septa. They also reported that these radiologic features could be categorized into four types based on which one was predominant: solid nodular, round GGO, alveolar interstitial and bronchovascular (12). Inoue et al. also indicated, based on the radiologic and pathological correlations, that IgG4-RLD could be interpreted as a sclerosing inflammatory lesion that is distributed along the intrapulmonary connective tissues, including the alveolar interstitium, the interlobular septa and the bronchovascular bundles $(12,13)$. In our patient, the pathological findings were consistent with IgG4-RLD that was characterized by the sclerosing inflammatory lesions along the intrapulmonary connective tissues. However, our case is unusual in that organizing pneumonia was concomitantly observed. Reflecting the pathological findings, the CT findings confirmed consolidation mainly around the bronchovascular bundles but the scans did not correspond to any of the four major types of CT features postulated by Inoue et al.

Although the etiology of IgG4-RD has not yet been elucidated, Th2 cytokines (IL-4, 5, and 13), regulatory $\mathrm{T}$ cells (Tregs) and Treg cytokines (IL-10 and transforming growth factor- $\beta$ ) are reportedly increased in these lesions. It has been reported that these factors may be associated with the pathogenesis of $\operatorname{IgG} 4-\mathrm{RD}(14,15)$. Organizing pneumonia is a frequently observed response pattern that is attributed to post-respiratory infection changes, drug-related tissue damage, radiation therapy, organ transplantation, occupational and environmental factors and rheumatological or connective 
tissue diseases, and that is also associated with underlying lung diseases, such as pulmonary abscess and lung cancer. It has also been reported that organizing pneumonia has occasionally been observed to complicate fibrosing lung diseases, such as idiopathic pulmonary fibrosis (IPF)/usual interstitial pneumonia (UIP) and nonspecific interstitial pneumonia (NSIP) (16). Given that IgG4-RD is a disease that is partly characterized by fibrosis, cases of IgG4-RLD which are complicated by organizing pneumonia may be less rare than previously thought.

Moreover, several cases presenting with the pathological features of both chronic eosinophilic pneumonia (CEP) and bronchiolitis obliterans with organizing pneumonia (BOOP) have been reported $(17,18)$. Some BOOP cases present with a few foci of organizing alveolar exudates that contain eosinophils surrounded by histiocytes. Because these foci are strongly reminiscent of a resolving eosinophilic abscess, a feature of CEP, Bartter et al. speculated that BOOP may develop partly as a consequence of the incomplete resolution of CEP (18). Interestingly, a lesion extremely similar to that reported by Bartter et al. was observed in our patient (Fig. 3C). It is believed that the pathogeneses of CEP and eosinophilic infiltration that are observed in IgG4-RD lesions are mediated via Th2 cytokines. As is the case with CEP complicated by organizing pneumonia, we speculate that IgG4-RLD may be complicated by organizing pneumonia as a consequence of the incomplete resolution of eosinophilic inflammation.

Based on the aforementioned observations, it is understandable that IgG4-RLD would be accompanied by organizing pneumonia. However, to our knowledge, there are only 2 case reports on IgG4-RD that were potentially complicated by a histologically-proven case of organizing pneumonia. These cases had autoimmune pancreatitis complicated by organizing pneumonia, and were reported by Duvic et al. (19) and Kobayashi et al. (20). In the case reported by Duvic et al., IgG4-positive cells were not histologically demonstrated. In the case reported by Kobayashi et al., the disease was also complicated by Sjogren's syndrome, and neither the $\mathrm{IgG}^{+} / \mathrm{IgG}^{+}$cell ratio nor the number of $\mathrm{IgG}^{+}$ plasma cells/HPF in lung biopsy samples was determined. It is therefore difficult to interpret the lung lesions in these cases.

Because IgG4-RLD is a recently designated disease entity, cases with IgG4-RLD alone, i.e., in which no extrapulmonary lesions were identified, may not have been correctly diagnosed. Although this case satisfied the present diagnostic criteria, it was difficult for us to diagnose because of the variations in his pathological findings. In the past, cases with prominent organizing pneumonia, such as our present patient, may have been misdiagnosed as having cryptogenic organizing pneumonia (COP) or CEP based on allergic predispositions, eosinophilia in the peripheral blood samples, the bronchoalveolar lavage fluid findings, etc. In the future, in order to elucidate the pathology of IgG4-RLD and establish the disease spectrum, it may be necessary to measure the serum IgG4 concentrations and to investigate histopathological features of IgG4-RD in those cases suspected to have COP or CEP, as well as to examine these cases in detail while considering the possibility of IgG4RLD.

The authors state that they have no Conflict of Interest (COI).

\section{Acknowledgement}

The authors would like to thank the staff of the Department of Clinical Laboratory, Saiseikai Yamagata Saisei Hospital for their clinical assistance.

\section{References}

1. Taniguchi T, Ko M, Seko S, et al. Interstitial pneumonia associated with autoimmune pancreatitis. Gut 53: 770-771, 2004.

2. Takato H, Yasui M, Ichikawa $Y$, et al. Nonspecific interstitial pneumonia with abundant IgG4-positive cells infiltration, which was thought as pulmonary involvement of IgG4-related autoimmune disease. Intern Med 47: 291-294, 2008.

3. Zen Y, Kitagawa $\mathrm{S}$, Minato $\mathrm{H}$, et al. IgG4-positive plasma cells in inflammatory pseudotumor (plasma cell granuloma) of the lung. Hum Pathol 36: 710-717, 2005.

4. Shigemitsu H, Koss MN. IgG4-related interstitial lung disease: a new and evolving concept. Curr Opin Pulm Med 15: 513-516, 2009.

5. Tsushima K, Tanabe $\mathrm{T}$, Yamamoto $\mathrm{H}$, et al. Pulmonary involvement of autoimmune pancreatitis. Eur J Clin Invest 39: 714-722, 2009.

6. Tanaka K, Nagata K, Tomii K, Imai Y. A case of isolated IgG4related interstitial pneumonia: a new consideration for the cause of idiopathic nonspecific interstitial pneumonia. Chest 142: 228-230, 2012.

7. Matsui S, Hebisawa A, Sakai F, et al. Immunoglobulin G4-related lung disease: clinicoradiological and pathological features. Respirology 18: 480-487, 2013.

8. Umehara H, Okazaki K, Masaki Y, et al. Comprehensive diagnostic criteria for IgG4-related disease (IgG4-RD), 2011. Mod Rheumatol 22: 21-30, 2012.

9. Sato Y, Kojima M, Takata K, et al. Systemic IgG4-related lymphadenopathy: a clinical and pathologic comparison to multicentric Castleman's disease. Mod Pathol 22: 589-599, 2009.

10. Deshpande V, Zen Y, Chan JK, et al. Consensus statement on the pathology of IgG4-related disease. Mod Pathol 25: 1181-1192, 2012.

11. Ikari J, Kojima M, Tomita K, et al. A case of IgG4-related lung disease associated with multicentric Castleman's disease and lung cancer. Intern Med 49: 1287-1291, 2010.

12. Inoue $D$, Zen $Y$, Abo $H$, et al. Immunoglobulin G4-related lung disease: CT findings with pathologic correlations. Radiology 251: 260-270, 2009.

13. Zen Y, Inoue D, Kitao A, et al. IgG4-related lung and pleural disease: a clinicopathologic study of 21 cases. Am J Surg Pathol 33: 1886-1893, 2009.

14. Detlefsen S, Sipos B, Zhao J, Drewes AM, Klöppel G. Autoimmune pancreatitis: expression and cellular source of profibrotic cytokines and their receptors. Am J Surg Pathol 32: 986-995, 2008.

15. Zen Y, Fujii T, Harada K, et al. Th2 and regulatory immune reactions are increased in immunoglobin G4-related sclerosing pancreatitis and cholangitis. Hepatology 45: 1538-1546, 2007.

16. Epler GR. Bronchiolitis obliterans organizing pneumonia, 25 years: a variety of causes, but what are the treatment options? Expert Rev Respir Med 5: 353-361, 2011. 
17. Carrington $\mathrm{CB}$, Addington WW, Goff AM, et al. Chronic eosinophilic pneumonia. N Engl J Med 280: 787-798, 1969.

18. Bartter T, Irwin RS, Nash G, Balikian JP, Hollingsworth HH. Idiopathic bronchiolitis obliterans organizing pneumonia with peripheral infiltrates on chest roentgenogram. Arch Intern Med 149: 273279, 1989.

19. Duvic C, Desrame J, Lévêque C, Nedelec G. Retroperitoneal fi- brosis, sclerosing pancreatitis and bronchiolitis obliterans with organizing pneumonia. Nephrol Dial Transplant 19: 2397-2399, 2004.

20. Kobayashi H, Shimokawaji T, Kanoh S, Motoyoshi K, Aida S. IgG4-positive pulmonary disease. J Thorac Imaging 22: 360-362, 2007.

(C) 2013 The Japanese Society of Internal Medicine http://www.naika.or.jp/imonline/index.html 